\section{Melanom: Langzeitdaten für BRAF-/MEK-Inhibition}

\section{Die Kombination der Kinaseinhibito- ren Dabrafenib und Trametinib war bereits in einer Zwischenanalyse der Dabrafenib-Monotherapie bei Pati- enten mit metastasiertem Melanom überlegen. Nun liegen die 3-Jahres- Daten vor.}

$\mathrm{n}$ die doppelblinde Phase-III-Studie COMBI-d waren 423 zuvor unbehandelte Patienten mit einem nicht resezierbaren Melanom im Stadium IIIC oder IV und einer BRAF-V600E/K-Mutation aufgenommen worden. Die Patienten erhielten randomisiert entweder 2-mal täglich $150 \mathrm{mg}$ des BRAF-Inhibitors Dabrafenib plus 1-mal täglich $2 \mathrm{mg}$ des MEK-Inhibitors Trametinib als Dauertherapie bis zur Progression oder aber
Dabrafenib plus Placebo. Primärer Endpunkt war das progressionsfreie Überleben (PFS); sekundäre Endpunkte waren das Gesamtüberleben (OS), die Gesamtansprechrate, die Ansprechdauer, die Sicherheit und die Pharmakokinetik.

Nach dem Daten-Cut-off im Februar 2016 zeigten sich bessere Ergebnisse für die Kombination. Damit wurden die Daten des 20 Monats-Follow-ups bestätigt. Nach 3 Jahren betrug die Rate für das PFS unter Dabrafenib plus Trametinib 22\% gegenüber $12 \%$ unter der Monotherapie, die Rate für das OS 44 versus $32 \%$, und das, obwohl 25 Patienten im Monotherapie-Arm nach der 20-Monats-Analyse auf die Kombinationstherapie gewechselt hatten.

Die Rate für das 3-Jahres-OS unter der Kombination erreichte $62 \%$ in der güns- tigsten Subgruppe (normaler Laktatdehydrogenase[LDH]-Spiegel und $<3$ von Metastasen betroffene Organe) gegenüber nur $25 \%$ in der ungünstigsten Subgruppe (erhöhte LDH-Spiegel). Das Sicherheitsprofil von Dabrafenib plus Trametinib war mit den bisherigen klinischen Beobachtungen konsistent; beim Langzeitgebrauch ergaben sich keine neuen Warnhinweise.

Fazit: Diese Daten zeigen, dass sich mit einer Erstlinienbehandlung aus $\mathrm{Da}$ brafenib plus Trametinib bei Patienten mit metastasiertem Melanom und BRAF-V600-Mutation ein langes, über 3 Jahre anhaltendes Überleben erreichen lässt.

Christina Berndt

Long GV et al. Dabrafenib plus trametinib versus dabrafenib monotherapy in patients with metastatic BRAF V600E/ K-mutant melanoma: long-term survival and safety analysis of a phase 3 study. Ann Oncol. 2017;28(7):1631-9.

\section{Melanom: Prognosefaktoren für Kinaseinhibitoren}

\section{Eine gepoolte Analyse von Langzeitdaten sollte weiteren Aufschluss über Prädiktoren für einen Langzeitnutzen von Melanompatienten mit BRAF-Mu- tation unter der Therapie mit Dabrafenib plus Trametinib bringen.} sen 3-Jahres-Daten von Patienten $(\mathrm{n}=563)$ aus den beiden randomisierten Phase-III-Studien COMBI-d und COMBI-v ein, die mit Dabrafenib plus Trame-

tinib behandelt wurden. Die Ergebnisse waren konsistent mit denen der individuellen Studien: Die Rate für das progressionsfreie Überleben (PFS) betrug nach 3 Jahren $23 \%$, die für das Gesamt-
In die vorliegende gepoolte Analyse flos-

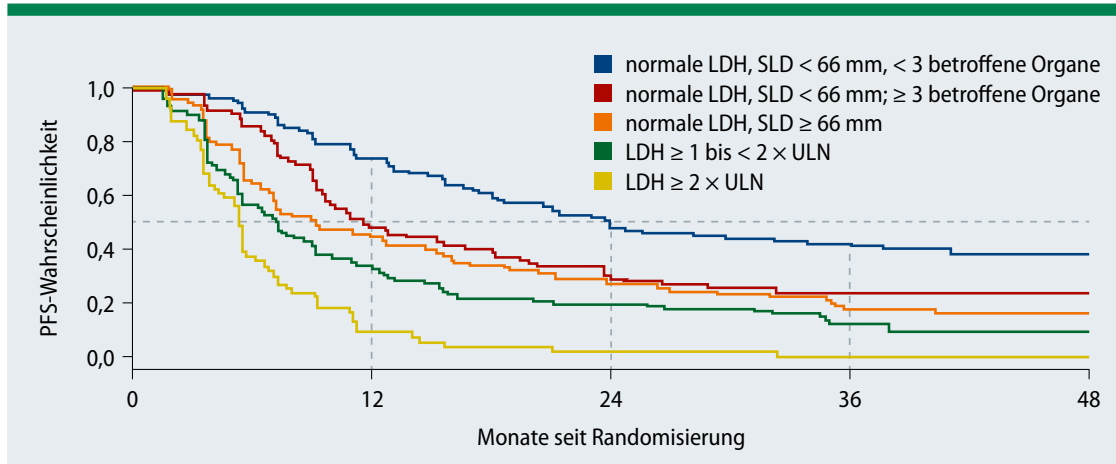

PFS = progressionsfreies Überleben; $L D H=$ Laktatdehydrogenase-Werte; SLD = Summe der Läsionsparameter; ULN = oberes Limit normaler Werte
Abb. 1: Das progressionsfreie Überleben der Melanompatienten variierte je nach Subgruppe stark. überleben (OS) 44\%. Die Laktatdehydrogenase (LDH)-Spiegel zu Therapiebeginn und die Zahl betroffener Organe blieben stark assoziiert mit PFS und OS und/oder prädiktiv dafür. Darüber hinaus wurde die Summe der Läsionsdurchmesser (SLD) zu Beginn der Therapie als Prädiktor für die Krankheitsprogression identifiziert.

In der günstigsten prognostischen Gruppe (normaler LDH-Spiegel, SLD < $66 \mathrm{~mm},<3$ betroffene Organe; $\mathrm{n}=$ 183/563 [33\%]), lag die PFS-Rate nach 3 Jahren bei $42 \%$ (Abb. 1). Die Zahl betroffener Organe zu Therapiebeginn war ebenfalls prädiktiv für das Ergebnis von Patienten mit einem PFS von $\geq$ 6 Monaten.

Fazit: Diese umfassendste Analyse von Phase-III-Daten zu einer BRAF/MEKInhibitor-Kombination beim Melanom zeigt, dass ein dauerhaftes Ansprechen über mindestens 3 Jahre bei Subgruppen von Patienten mit BRAF-mutiertem Melanom möglich ist. Christina Berndt

Schadendorf D et al. Three-year pooled analysis of factors associated with clinical outcomes across dabrafenib and trametinib combination therapy phase 3 randomised trials. Eur J Cancer. 2017; 82: 45-55. 